\title{
Disease-modifying therapies and SARS-CoV-2 vaccination in multiple sclerosis: an expert consensus
}

\author{
Diego Centonze ${ }^{1,2} \cdot$ Maria A. Rocca ${ }^{3,6,7} \cdot$ Claudio Gasperini $^{8} \cdot$ Ludwig Kappos $^{9} \cdot$ Hans-Peter Hartung ${ }^{10,11,12}$. \\ Melinda Magyari $^{13} \cdot$ Celia Oreja-Guevara $^{14,15} \cdot$ Maria Trojano $^{16} \cdot$ Heinz Wiend $^{17} \cdot$ Massimo Filippi $^{3,4,5,6,7}$ (1)
}

Received: 17 February 2021 / Revised: 1 April 2021 / Accepted: 3 April 2021 / Published online: 12 April 2021

(C) The Author(s) 2021

\begin{abstract}
Coronavirus disease (COVID-19) appeared in December 2019 in the Chinese city of Wuhan and has quickly become a global pandemic. The disease is caused by the severe acute respiratory syndrome coronavirus type-2 (SARS-CoV-2), an RNA beta coronavirus phylogenetically similar to SARS coronavirus. To date, more than 132 million cases of COVID19 have been recorded in the world, of which over 2.8 million were fatal (https://coronavirus.jhu.edu/map.html). A huge vaccination campaign has started around the world since the end of 2020. The availability of vaccines has raised some concerns among neurologists regarding the safety and efficacy of vaccination in patients with multiple sclerosis (MS) taking immunomodulatory or immunosuppressive therapies.
\end{abstract}

Keywords Multiple sclerosis $\cdot$ Disease-modifying treatments $\cdot$ Vaccine $\cdot$ COVID-19 pandemic

Massimo Filippi

filippi.massimo@hsr.it

1 Department of Systems Medicine, Tor Vergata University, Rome, Italy

2 Unit of Neurology, IRCCS Neuromed, Pozzilli (IS), Italy

3 MS Center and Neurology Unit, IRCCS San Raffaele Scientific Institute, Via Olgettina, 60, 20132 Milan, Italy

4 Neurorehabilitation Unit, IRCCS San Raffaele Scientific Institute, Milan, Italy

5 Neurophysiology Service, IRCCS San Raffaele Scientific Institute, Milan, Italy

6 Neuroimaging Research Unit, Division of Neuroscience, IRCCS San Raffaele Scientific Institute, Milan, Italy

7 Vita-Salute San Raffaele University, Milan, Italy

8 Department of Neurosciences, San Camillo Forlanini Hospital, Rome, Italy

9 MS Center and Research Center for Clinical Neuroimmunology and Neuroscience Basel (RC2NB), Departments of Medicine, Clinical Research and Biomedicine and Biomedical Engineering, University Hospital and University of Basel, Basel, Switzerland
10 Department of Neurology, Medical Faculty, Heinrich-Heine University, University Hospital Duesseldorf, Düsseldorf, Germany

11 Brain and Mind Centre, University of Sydney, Sydney, Australia

12 Department of Neurology, Medical University of Vienna, Wien, Austria

13 Danish Multiple Sclerosis Center, Department of Neurology, Copenhagen University Hospital, Rigshospitalet, Copenhagen, Denmark

14 Department of Neurology, Hospital Clínico San Carlos, IdISSC, Madrid, Spain

15 Departamento de Medicina, Facultad de Medicina, Universidad Complutense de Madrid (UCM), Madrid, Spain

16 Neurology and Neurophysiopathology Unit, Department of Basic Medical Sciences, Neuroscience and Sense Organs, University of Bari “Aldo Moro", Bari, Italy

17 Department of Neurology, University Hospital Münster, Münster, Germany 


\section{COVID-19}

The clinical course of COVID-19 is characterized by three different phases. In the first phase of the disease, the patient is asymptomatic or has mild flu-like symptoms characterized by fatigue, high temperature and dry cough. In this phase the active virus replication takes place and lymphopenia may be present. As the disease progresses, a typical viral pneumonia evolves detectable typically on chest computed tomography as pneumonic infiltrates, so-called ground-glass opacities. The most severe phase of the disease is characterized by a hyper-inflammatory state sustained by the high production of proinflammatory cytokines, the so-called cytokine storm. This leads to sepsis and disseminated intravasal coagulation which may manifest with both multiorgan failure and fatal outcome [1].

The main entry route of SARS-CoV-2 involves the interaction between the viral spike protein and the angiotensin-converting enzyme-related carboxypeptidase 2 (ACE2) expressed by the host cells [2]. The exact mechanisms of antigen presentation and consequent activation of the innate and adaptive immune response are not completely clear. The activation of innate immunity seems to achieve the strong cytopathic properties of virus, following the release of damage-associated molecular patterns and pathogen-associated molecular patterns, giving rise to an excessive production of proinflammatory cytokines. Confirming this hypothesis, a marked increase in circulating inflammatory cytokines, such as interleukin (IL)-1 $\beta$, IL-6, IL-7 and TNF- $\alpha$, is observed in patients with more severe symptoms. Moreover, the increased production of such molecules, so-called cytokine storm, correlates with a more rapid progression to acute respiratory distress syndrome [3]. To date, the exact cellular source of the cytokines is not clear. Two scenarios are considered possible and not mutually exclusive. The first is that cells of the innate immunity, directly stimulated by the viral infection, produce inflammatory mediators. The alternative hypothesis holds that the hyperactivation of innate immunity is secondary to hyper-activated T cells [4]. The affection of the central nervous system (CNS) is not uncommon in COVID-19, anosmia, stroke and encephalopathy being the most prominent manifestations [5]. Mechanisms are not yet entirely clear, but Neuro-COVID seems to be associated with an exhaustion of $\mathrm{T}$ cells and dedifferentiation of monocytes in the cerebrospinal fluid [6].

As with all viral infections, adaptive immunity is also important in countering SARS-CoV-2 infection. CD4 central memory and CD8 effector memory cells represent the cellular subsets most active against viral SARS-CoV-2 proteins [7]. Dysregulation of T-cell immunity is likely to contribute to disease severity, low T-regulatory subsets and expansion of pro-inflammatory GM-CSF+CD4+ and IL-6+ CD4+ T cells have been detected in patients with more severe disease [4].

Regarding humoral immunity, the appearance of protective antibodies occurs 7-14 days after the onset of disease. However, it should be considered that the presence of a high IgG response is associated with a more severe disease course, suggesting a detrimental pathogenic antibodydependent response in the same cases [8].

\section{Disease-modifyng therapies and COVID-19}

Almost all MS patients take immunomodulatory or immunosuppressive drugs to lessen disease activity, severity and to prevent or slow disease progression. Therefore, the current COVID-19 pandemic requires us to understand whether these therapies can increase the risk of infection or worsen the clinical course of the disease [9]. Currently, available evidence does not suggest that MS patients per se develop more severe COVID-19 [10,11]. A recent international study conducted on a database of over 30,000 patients with MS highlighted how the main risk factors were related to pre-existing clinical conditions, such as comorbidity score $\geq 1$, body mass index $\geq 30$, and Black/ African ancestry, comparable to those seen in the general population [12].

Interferon- $\beta$ therapies (IFNs) represent the first diseasemodifying therapy (DMT) approved for the treatment of MS. Their mechanism of action is pleiotropic, involving the shift of the balance toward the anti-inflammatory Th- 2 cells and inhibition of T-cell migration as a result of blockade of metalloproteases and adhesion molecules. The activation of the IFN pathway leads to the induction of several genes, so-called IFN-stimulated genes, many of which interfere with the synthesis of viral proteins [13]. Preliminary data seem to show a certain sensitivity of SARS-CoV-2 to treatment with type-I IFNs, as in vitro IFN I causes an attenuation of SARS-CoV-2, but not SARS-CoV, replication [14]. Another study showed that early IFN therapy is associated with favorable clinical responses in COVID-19 patients [15]. Overall, treatment with IFNs does not appear to increase the risk of infection or worsen the disease course $[16,17]$, while many studies demonstrate that these molecules reduce the risk of SARSCoV-2 infection in treated MS patients [12].

Glatiramer acetate (GA) is a synthetic polypeptide containing four naturally occurring amino acids (L-glutamic acid, L-lysine, L-alanine and L-tyrosine), like the myelin basic protein. Although its precise mechanism of action is still unknown, GA has been reported to induce a shift from Th1 to Th2 responses, with an increase in T-regulatory and 
down-regulation of both Th1 and Th17 cells. The extensive data available from clinical practice do not show an enhanced infective risk in patients taking GA [18], and some studies seem to prove a lower incidence of positivity to SARS-CoV-2 in patients treated with this drug [12]. Hence no greater risk of infection is expected nor any impact on the clinical course of COVID-19 disease.

Fingolimod, the first oral drug approved for MS treatment, is a sphingosine-1-phosphate receptor modulator. It blocks only central memory $\mathrm{T}$ cells in lymph nodes, without compromising the circulation of effector memory $\mathrm{T}$ cells, and for this reason it generally does not affect the ability to respond to infections [19]. It is still unclear whether fingolimod can negatively affect SARSCoV-2 infection, however, preclinical data show that sphingosine-1-phosphate receptor modulating drugs reduce the inflammatory pulmonary infiltrate during influenza infection and enhance endothelial barrier function [20].

Teriflunomide interferes with de novo pyrimidine synthesis by specific inhibition of the mitochondrial enzyme dihydro-orotate dehydrogenase (DHODH), an enzyme highly expressed in proliferating lymphocytes. By interfering with pyrimidines synthesis, teriflunomide also inhibits viral replication, as confirmed by in vitro data [21]. Current data from case series and retrospective studies show that severe disease course was observed in none of 79 patients treated with teriflunomide and infected with SARS-CoV-2 [17, 22-24]. Moreover, teriflunomide is even assumed to have possibly beneficial effects because of its antiviral mechanism, and a DHODH inhibiting principle is currently under investigation to prevent COVID-19 morbidity and mortality [25].

Although the therapeutic mechanism of action of dimethylfumarate in MS has not been fully elucidated, it is thought to stimulate Nrf2-pathways with consequent anti-oxidative, anti-inflammatory and cytoprotective effects. Experimental data show that enhancement of the NRF2-pathway reduces macrophage-mediated alveolar damage and induces the expression of antioxidant genes during influenza [26]. Moreover, a recent study demonstrated that dimethylfumarate inhibits SARS-CoV-2 replication and the expression of associated inflammatory genes [27]. Therefore, dimethyl fumarate could have a beneficial anti-inflammatory and cytoprotective action in SARS-CoV-2 infection. Small case series suggest the safety of dimethylfumarate in patients with COVID-19 and normal lymphocyte count, although in the case of lymphopenia a more severe infection cannot be excluded [28].

Natalizumab is a monoclonal antibody that binds to alpha-4 integrin and thereby prevents leukocytes from crossing the blood-brain-barrier. Except for progressive multifocal leukoencephalopathy, natalizumab does not significantly increase the risk of infections [29]. Therefore, it is not expected to increase COVID-19 severity [30]. Most neurological complications during COVID-19 seem to be secondary to indirect mechanisms as cytokine storm, hypercoagulability, multiorgan failure, dysmetabolic states, para- and post-infectious autoimmunity [31]. However, the possible direct invasion of CNS by SARS-Cov-2, a rare but noteworthy condition, must be considered. In this case, the reduction of immunosurveillance in CNS caused by natalizumab could in principle cause severe neurological involvement and sequelae.

Recently, the North American Registry of MS patients described a reduced risk of ICU admission and ventilation in patients treated with fumarate and natalizumab compared to untreated patients [11].

Alemtuzumab is a monoclonal antibody that binds the CD52 glycoprotein and induces antibody-dependent and complement-mediated cytotoxicity. There is an increased infection risk following alemtuzumab therapy, which is dependent on the time of administration of the drug [32]. It is well known that the percentage of infectious complications during therapy with alemtuzumab is closely related to the lymphocyte count and in particular to the lymphopenia that the drug itself induces. Their incidence is, in fact, higher during the first month after drug infusion, and they mostly consist of mild upper respiratory and urinary tract infections, and very rarely systemic infections such as gastroenteritis, diffuse pneumonia and sepsis [33-35]. However, the predominant involvement of adaptive immunity accounts for the low rate of life-threatening infections [36]. The mild disease of the few reported cases of COVID-19 in patients treated with alemtuzumab suggests a prevalent beneficial anti-inflammatory effect of the drug [37-39].

Anti CD20 monoclonal antibodies induce cell lysis by antibody-dependent and complement-mediated cytotoxicity. In addition to B-cell specific subsets of $\mathrm{CD} 20+, \mathrm{T}$ cells are also targeted by the drug. B-depletion has an effect not only on humoral immunity but also on T-mediated responses. Indeed $\mathrm{B}$ cells are necessary for maintaining naive $\mathrm{CD} 4+$ and CD8+ T-cell homeostasis [40]. It has been reported that antiCD20 monoclonal antibodies polarize innate immunity cells in a less inflammatory way, thus potentially mitigating the inflammatory response to the virus, and essentially preventing the evolution to severe forms of the disease [41]. It is still unclear, however, whether anti CD20 antibodies elevate the risk of SARS-CoV-2 infection, as reported in a recent clinical study [12]. Duration of exposure might play a role, as suggested by the North American Registry, which found an increased risk of hospitalization in patients treated with rituximab, but not in those treated with ocrelizumab [11].

Probably, the increased rate of infection could be explained by the fact that anti-CD20 antibodies induce an inability to produce, especially close to the drug infusion, an adequate humoral response against the pathogen [42]. 
The possibility of developing iatrogenic hypogammaglobulinemia should also be considered, which, in addition to increasing the risk of infection, tends to positively affect the rate of reinfection, hindering the development of anti SARSCoV-2 antibodies after infection with COVID-19 in patients treated with ocrelizumab [43]. Notably, the risk factors that can influence severe COVID-19 disease courses in patients treated with ocrelizumab are the same as in the general population. At the same time, fatal cases of SARS-CoV-2 infection in MS patients treated with ocrelizumab seem to occur at a higher percentage than those occurring during treatment with other approved DMTs [44]. This is probably due to a higher prevalence of Sars-CoV-2 infections in MS patients treated with anti-CD20 compared to other drugs. A recent study conducted in patients with COVID-19 and rheumatic diseases treated with rituximab, another anti-CD-20 drug, demonstrated an increase in the rate of hospitalization in intensive care units and an increase in mortality rate compared to patients treated with other antirheumatic drugs [45]. This evidence seems to be confirmed after correction for possible confounding factors, although, by admission of the authors themselves, patients treated with rituximab generally have a poor response to traditional first-line therapies, together with extra-articular manifestations of disease, chronic use of corticosteroids, and cardiovascular and metabolic risk factors similar to the general population affected by severe forms of COVID-19 disease.

Cladribine is a purine analog that interferes with DNA synthesis. It induces a prolonged lymphocyte depletion more evident for B lymphocytes [46]. Phase-III studies have shown a low infection risk in cladribine-treated patients which may be explained by the fact that patients in treatment with this drug rarely develop severe lymphopenia with lymphocyte ratio less than 500 cells/ml [47]. Since the drug has been introduced only a couple of years ago, few data are available to date. However, given the low impact on innate immunity of cladribine, a higher risk of developing severe COVID-19 is not expected.

\section{COVID-19 vaccines}

Although some drugs cause reduced disease duration and mortality, currently there is no specific therapy effective in all cases of COVID-19 [48]. Therefore, vaccination is the most effective way to counteract the pandemic. The complete genomic sequencing of SARS-CoV-2, available from January 2020, allowed the rapid development of several new-generation vaccines. SARS-CoV-2 is a single-stranded positive-sense RNA virus composed of four main structural proteins: spike protein (S), envelope protein $(\mathrm{E})$, membrane protein $(\mathrm{M})$ and nucleocapsid protein $(\mathrm{N})$. The $\mathrm{S}$ protein, located at the outer surface of the virus particles, binds to
ACE2 on the cell surface allowing receptor-mediated endocytosis of the virus [49]. As protein $\mathrm{S}$ is crucial for the virus to enter the cell, many vaccines use this protein as antigen.

To date (March 2021), 3 vaccines have been approved by EMA in the EU. Two are mRNA-vaccines encoding protein $\mathrm{S}$. They do not contain live materials so they are easier to synthesize and do not have the risk of disease transmission. As mRNA molecules have low transfection efficacy, lipid nanoparticles are used to incorporate them to augment transfection capacity [50]. Once phagocytosed by a cell, lipid nanoparticles are exposed to a low-pH environment in the endosome and the RNA-condensing lipid can pierce the endosome and allow the mRNA molecule to be released in the cytosol.

These vaccines are administered intramuscularly in two administrations 21 days (Pfizer-BioNTech), or 28 days (Moderna) apart.

The third vaccine approved (produced by AstraZeneca) and widely administered in the UK and in many countries of the world is an adenoviral vector-based vaccine. In this vaccine, the double-stranded DNA encoding the coronavirus spike protein antigen is cloned into a viral vector that lacks the ability to reproduce. The viral vector, mimicking a viral infection disease state, produces strong cellular immune responses [51]. Two intramuscular injections are administered 4-12 weeks apart. Other new generation vaccines under study are recombinant protein vaccine, bacterial vector-based vaccine, plasmid DNA vaccine and trained immunity-based vaccine. Traditional inactivated vaccines are also among the candidate vaccines against SARS-CoV-2 (for regular updates please look at London school of hygiene $\&$ Tropical medicine-Vaccine tracker https://vac-lshtm. shinyapps.io/ncov_vaccine_landscape/\#).

\section{Vaccines and DMTs}

The development of protective immunity over time mainly requires the correct functionality of adaptive immunity played by B and T lymphocytes. Stimulation of B and T lymphocytes by specific antigens leads to their clonal expansion with the formation of memory clones. Memory cells after a re-exposure to the same antigen proliferate rapidly transforming themselves into effector cells. B cells upon activation differentiate into plasma cells which first produce IgM class antibodies and later IgG antibodies.

Safety and efficacy of vaccines against various pathogens in patients with MS are now well established. Several studies have shown that there is no difference in vaccine responses between MS patients and healthy subjects [52]. Conversely, the evaluation of safety and effectiveness of vaccines in MS patients taking DMT is more complex. 
Several studies demonstrate that IFNs do not compromise the efficacy of vaccines. No significant differences between patients treated with IFNs and healthy subjects were found in the degree and duration of humoral immunity. Cellular immune response, as measured by the frequency of IFNgamma secreting $\mathrm{T}$ cells in response to influenza antigen, was also similar [53]. Even duration of protective antibody titer against influenza was comparable between patients treated with IFNs and healthy subjects [54]. To date, overall available data demonstrate adequate immune responses to sundry vaccines in MS patients treated with IFNs.

An observational study comparing the immune response to inactivated influenza vaccine between patients treated with GA and healthy subjects showed similar seroprotection in the two groups. The authors detected a lower response in the treatment group, however, the difference was not statistically significant [55]. An efficient immune response was confirmed by a recent prospective study in which patients on GA demonstrated post-influenza vaccination seroprotection rates close to those of the comparator beta-interferon treated group [56].

Teriflunomide showed a modestly diminished rate of immune response to vaccines, however, it does not appear to compromise the achievement of seroprotective antibody levels. Patients treated with teriflunomide generated effective immune responses to seasonal influenza vaccination [57]. Antibody responses to rabies vaccine neoantigen and delayed hypersensitivity to candida albicans, trichophyton and tuberculin were shown to be safe and effective in subjects treated with teriflunomide, although the ratios of postvaccination to pre-vaccination geometric mean titers was lower in the treated versus the placebo group [58].

An observational study evaluating vaccine responses in patients taking dimethyl fumarate and IFNs revealed comparable vaccine response in both groups [59]. Based on these data and considering the mechanism of action of the drug, a negative effect on vaccinations is not expected.

A blinded, randomized, multicentre, placebo-controlled study of responses to seasonal influenza vaccine and tetanus toxoid booster was conducted in patients receiving fingolimod. Although many patients have shown to develop a valid immune response, the proportion that achieved seroprotective titers was lower in the treatment group than healthy subjects [60]. The reduced response to influenza vaccine during treatment with fingolimod was also confirmed by a further prospective study [55]. Similar results were also observed for the S1P receptor modulator siponimod [61].

A similar immune response to diphtheria-tetanus toxoid and neoantigen Keyhole limpet hemocyanin (KLH) was detected in patients treated with natalizumab and healthy subjects [62]. Conversely, a real-life study and a prospective study evaluating, respectively, H1N1 [54] and seasonal influenza vaccination [55] have provided evidence that an inadequate response to the immunization may occur in some patients treated with natalizumab.

The only small study evaluating the effect of alemtuzumab on 3 vaccines (diphtheria, tetanus, and poliomyelitis vaccine, haemophilus influenzae type $\mathrm{B}$ and meningococcal group $\mathrm{C}$ conjugate vaccine, and pneumococcal polysaccharide vaccine) demonstrated normal responses, though somewhat blunted for vaccinations within 6 months from last infusion [63]. However, in light of the limited data available, it is recommended to complete vaccinations at least 6 weeks before starting alemtuzumab. The timing of vaccination is further controversial if therapy has been already started. In this case, it is reasonable to wait 3-6 months after the last dose. In fact, after druginduced depletion, B cells usually return at basal levels at 3-6 months, although 12 months are generally requested to switch to a mature phenotype. Moreover, it should be considered that cellular $\mathrm{T}$ depletion tends to persist much longer (about 30 months for CD8+ cells and 60 months for CD4+ cells) [64].

The VELOCE trial investigated the effect of ocrelizumab on different vaccinations. Humoral response to inactivated vaccines studied was detected, although attenuated [65]. Therefore, as some patients may not develop an effective immunization during B-cell depletion it may be useful to delay the first course of ocrelizumab and receive the vaccine at least 6 weeks before infusion. If the risk/benefit ratio supports the need for an urgent (re)treatment, an individualized strategy should be assessed for each patient. For example, if a normal ocrelizumab treatment regimen is scheduled (dosing interval of 6 months) it could be reasonable to administer the vaccine at least 3 months after the last infusion. Indeed, for mRNA approved SARS-CoV-2 vaccines a 3-week interval between the first and second dose is necessary, then further 4-6 weeks are needed to develop an adequate immunization before ocrelizumab re-administration.

To date, no relevant studies on maintenance and/or generation of immune responses have been reported in MS patients on oral cladribine. Considering the complex mechanism of action of cladribine, investigating immune response to vaccines at different timepoints after treatment could be useful. Following cladribine administration, a rapid depletion of both $\mathrm{T}$ and $\mathrm{B}$ peripherally circulating lymphocytes is observed, with a mean nadir at 13-24 weeks [46]. Several studies are currently ongoing to test humoral and cell-mediated immune responses during vaccination in patients treated with cladribine. Among these, CLOCK-MS, a 24-month, open-label, randomized, multicenter phase-IV research study identified antibody titers after administration of influenza vaccine in patients treated with cladribine. In particular, increased antibody titers after 4 weeks from vaccination were demonstrated [66]. Furthermore, in MSMagnify study, aimed at determining the onset of cladribine 
effect in highly active RRMS patients, it was observed that seroprotection or increased seasonal influenza titers occurred in treated patients who were vaccinated early (month 1.5 to 6 of year 1, and month 1 to 4.5 of year 2 of treatment) or late (month 8.5 to 10.5 of year 1), and that seroprotection was maintained irrespective of total lymphocyte count [67]. Finally, the CLARITY clinical trial, conducted in patients treated with oral cladribine $(3.5 \mathrm{mg} / \mathrm{kg})$ vs placebo, has not documented adverse events associated with the administration of vaccines [68]. So it could be reasonable to wait at least 4 weeks after the last course of therapy or, in case of lymphopenia, to wait until the recovery of lymphocyte count.

In summary, currently available data show that vaccinations do not exacerbate MS, provoke a relapse, or prevent DMTs from being effective. Inactivated vaccines are considered safe in patients treated with any disease-modifying drugs, although in some cases may be less effective. In any case, it is not recommended to discontinue or modify DMTs to improve vaccine efficacy, as the risk of disease reactivation and progression outweighs the potential benefit [9]. At the same time, a reduced response is likely to be better than none. Knowledge of vaccine responses on DMTs and a careful risk/benefit assessment is mandatory. If at all possible, vaccinations clearly should be recommended and administered to people with MS.

Funding The authors received no funding for this study.

\section{Declarations}

Conflicts of interest D. Centonze is an Advisory Board member of Almirall, Bayer Schering, Biogen, GW Pharmaceuticals, Merck Serono, Novartis, Roche, Sanofi-Genzyme, and Teva and received honoraria for speaking or consultation fees from Almirall, Bayer Schering, Biogen, GW Pharmaceuticals, Merck Serono, Novartis, Roche, Sanofi-Genzyme, and Teva. He is also the principal investigator in clinical trials for Bayer Schering, Biogen, Merck Serono, Mitsubishi, Novartis, Roche, Sanofi-Genzyme, and Teva. His preclinical and clinical research was supported by grants from Bayer Schering, Biogen Idec, Celgene, Merck Serono, Novartis, Roche, Sanofi-Genzyme and Teva. M.A. Rocca received speakers honoraria from Bayer, Biogen, Bristol Myers Squibb, Celgene, Genzyme, Merck Serono, Novartis, Roche, and Teva, and receives research support from the Italian Ministry of Health, MS Society of Canada and Fondazione Italiana Sclerosi Multipla. C. Gasperini has received funding for an invited speaker or travel expenses for attending meetings from Biogen, Genzyme, Merck Serono, Novartis, Roche, Sanofi, and Teva. L. Kappos' institution (University Hospital Basel) has received in the last 3 years and used exclusively for research support: steering committee, advisory board, and consultancy fees (Actelion, Bayer Health-Care, Biogen, Genzyme, Merck KGaA [Darmstadt, Ger-many], Novartis, Pfizer, Sanofi, Santhera, Teva); speaker fees (Bayer HealthCare, Biogen, Merck KGaA [Darmstadt, Germany], Novartis, Sanofi, and Teva); support of educational activities (Bayer HealthCare, Biogen, CSL Behring, Genzyme, Merck KGaA [Darmstadt, Germany], Novartis, Sanofi, and Teva); license fees for Neurostatus products; and grants (Bayer HealthCare, Biogen, European Union, Innoswiss, Merck KGaA [Darmstadt, Ger- many], Novartis, Roche, Swiss MS Society, and Swiss National Research Foundation). H.-P. Hartung has received fees for consulting, speaking, and serving on steering committees from Bayer HealthCare, Biogen Idec, Celgene BMS, CSL Behring, GeNeuro, Genzyme, MedDay, MedImmune, Merck Serono, Novartis, Roche, Sanofi, TG Therapeutics, and VielaBio with approval by the Rector of Heinrich Heine University Düsseldorf. M. Magyari has served on scientific advisory board for Biogen, Sanofi, Roche, Novartis, Merck, Abbvie, Alexion, has received honoraria for lecturing from Biogen, Merck, Novartis, Sanofi, has received research support and support for congress participation from Biogen, Genzyme, Roche, Merck, Novartis. C. Oreja-Guevara has received speaker and consultation fees from Biogen Idec, Celgene, Sanofi-Genzyme, Novartis, Roche, Merck, and Teva. M. Trojano received speaker's honoraria, consulting fees, honoraria in advisory boards, support for attendance of scientific meetings from Meck Serono, Biogen, Novartis, Teva, Roche and Sanofi Genzyme. $\mathrm{H}$. Wiendl received speaker's honoraria, consulting fees, honoraria in advisory boards, support for attendance of scientific meetings from Actelion, Alexion, Biogen, Cognomed, Evgen, F. Hoffmann-La Roche Ltd., GemeinnützigeHertie-Stiftung, Genzyme,IGES, Johnson \& Johnson,MedDay Pharmaceuticals, Merck Serono, Novartis, Roche Pharma AG, and Sanofi-Aventis, the Swiss Multiple Sclerosis Society. TEVA, and WebMD Global. His research is funded by the German Ministry for Education and Research (BMBF), Deutsche Forschungsgemeinschaft (DFG), Else Kröner Fresenius Foundation, Fresenius Foundation, the European Union, Hertie Foundation, NRW Ministry of Education and Research, Interdisciplinary Center for Clinical Studies (IZKF) Muenster, Biogen, GlaxoSmithKline, Roche Pharma, andSanofi-Genzyme. M. Filippi is Editor-in-Chief of the Journal of Neurology and Associate Editor of Human Brain Mapping; received compensation for consulting services and/or speaking activities from Almiral, Alexion, Bayer, Biogen, Celgene, Eli Lilly, Genzyme, MerckSerono, Novartis, Roche, Sanofi, Takeda, and Teva Pharmaceutical Industries; and receives research support from Biogen Idec, MerckSerono, Novartis, Roche, Teva Pharmaceutical Industries, Italian Ministry of Health, Fondazione ItalianaSclerosiMultipla, and ARiSLA (Fondazione Italiana di Ricerca per la SLA).

Ethics approval Not applicable.

Open Access This article is licensed under a Creative Commons Attribution 4.0 International License, which permits use, sharing, adaptation, distribution and reproduction in any medium or format, as long as you give appropriate credit to the original author(s) and the source, provide a link to the Creative Commons licence, and indicate if changes were made. The images or other third party material in this article are included in the article's Creative Commons licence, unless indicated otherwise in a credit line to the material. If material is not included in the article's Creative Commons licence and your intended use is not permitted by statutory regulation or exceeds the permitted use, you will need to obtain permission directly from the copyright holder. To view a copy of this licence, visit http://creativecommons.org/licenses/by/4.0/.

\section{References}

1. Siddiqi HK, Mehra MR (2020) COVID-19 illness in native and immunosuppressed states: a clinical-therapeutic staging proposal. J Heart Lung Transplant. https://doi.org/10.1016/j.healun.2020. 03.012

2. Hoffmann M et al (2020) SARS-CoV-2 cell entry depends on ACE2 and TMPRSS2 and is blocked by a clinically proven protease inhibitor. Cell. https://doi.org/10.1016/j.cell.2020.02.052 
3. Huang C et al (2020) Clinical features of patients infected with 2019 novel coronavirus in Wuhan, China. Lancet. https://doi.org/ 10.1016/S0140-6736(20)30183-5

4. Zhou Y et al (2020) Pathogenic T-cells and inflammatory monocytes incite inflammatory storms in severe COVID-19 patients. Natl Sci Rev. https://doi.org/10.1093/nsr/nwaa041

5. Solomon T (2021) Neurological infection with SARS-CoV-2the story so far. Nat Rev Neurol. https://doi.org/10.1038/ s41582-020-00453-w

6. Heming $\mathrm{M}$ et al (2021) Neurological manifestations of COVID19 feature $\mathrm{T}$ cell exhaustion and dedifferentiated monocytes in cerebrospinal fluid. Immunity. https://doi.org/10.1016/j.immuni. 2020.12.011

7. Weiskopf D et al (2020) Phenotype and kinetics of SARS-CoV2 -specific $\mathrm{T}$ cells in COVID-19 patients with acute respiratory distress syndrome. Sci Immunol. https://doi.org/10.1126/SCIIM MUNOL.ABD2071

8. Liu L et al (2019) Anti-spike IgG causes severe acute lung injury by skewing macrophage responses during acute SARS-CoV infection. JCI insight. https://doi.org/10.1172/jci.insight.123158

9. Korsukewitz C, Reddel SW, Bar-Or A, Wiendl H (2020) Neurological immunotherapy in the era of COVID-19-looking for consensus in the literature. Nat Rev Neurol. https://doi.org/10. $1038 / \mathrm{s} 41582-020-0385-8$

10. Louapre $\mathrm{C}$ et al (2020) Clinical characteristics and outcomes in patients with coronavirus disease 2019 and multiple sclerosis. JAMA Neurol. https://doi.org/10.1001/jamaneurol.2020.2581

11. Salter A et al (2021) Outcomes and risk factors associated with SARS-CoV-2 Infection in a North American registry of patients with multiple sclerosis. JAMA Neurol. https://doi.org/10.1001/ jamaneurol.2021.0688

12. Reder AT et al (2021) COVID-19 in patients with multiple sclerosis: associations with disease-modifying therapies. CNS Drugs. https://doi.org/10.1007/s40263-021-00804-1

13. Schoggins JW (2019) Interferon-stimulated genes: what do they all do? Annu Rev Virol. https://doi.org/10.1146/annurev-virol ogy-092818-015756

14. Lokugamage KG et al (2020) Type I interferon susceptibility distinguishes SARS-CoV-2 from SARS-CoV. J Virol. https://doi.org/ 10.1128/jvi.01410-20

15. Wang $\mathrm{N}$ et al (2020) Retrospective multicenter cohort study shows early interferon therapy is associated with favorable clinical responses in COVID-19 patients. Cell Host Microbe. https:// doi.org/10.1016/j.chom.2020.07.005

16. Sharifian-Dorche $M$ et al (2021) COVID-19 and disease-modifying therapies in patients with demyelinating diseases of the central nervous system: a systematic review. Mult Scler Relat Disord. https://doi.org/10.1016/j.msard.2021.102800

17. Sormani MP et al (2021) Disease-modifying therapies and coronavirus disease 2019 severity in multiple sclerosis. Ann Neurol. https://doi.org/10.1002/ana.26028

18. Luna $\mathrm{G}$ et al (2020) Infection risks among patients with multiple sclerosis treated with Fingolimod, Natalizumab, Rituximab, and injectable therapies. JAMA Neurol. https://doi.org/10.1001/jaman eurol.2019.3365

19. Pinschewer DD, Brinkmann V, Merkler D (2011) Impact of sphingosine 1-phosphate modulation on immune outcomes. Neurology. https://doi.org/10.1212/WNL.0b013e31820d9596

20. Walsh KB et al (2011) Suppression of cytokine storm with a sphingosine analog provides protection against pathogenic influenza virus. Proc Natl Acad Sci U S A. https://doi.org/10.1073/ pnas. 1107024108

21. Xiong $\mathrm{R}$ et al (2020) Novel and potent inhibitors targeting DHODH are broad-spectrum antivirals against RNA viruses including newly-emerged coronavirus SARS-CoV-2. Protein Cell. https://doi.org/10.1007/s13238-020-00768-w
22. Maghzi AH et al (2020) COVID-19 in teriflunomide-treated patients with multiple sclerosis. J Neurol. https://doi.org/10.1007/ s00415-020-09944-8

23. Mantero V et al (2020) Mild COVID-19 infection in a group of teriflunomide-treated patients with multiple sclerosis. J Neurol. https://doi.org/10.1007/s00415-020-10196-9

24. Capone F, Motolese F, Luce T, Rossi M, Magliozzi A, Di Lazzaro V (2021) COVID-19 in teriflunomide-treated patients with multiple sclerosis: a case report and literature review. Mult Scler Relat Disord. https://doi.org/10.1016/j.msard.2020.102734

25. Hahn F et al (2020) IMU-838, a developmental DHODH inhibitor in phase II for autoimmune disease, shows Anti-SARS-CoV-2 and broad-spectrum antiviral efficacy in vitro. Viruses. https://doi.org/ $10.3390 / \mathrm{v} 12121394$

26. Kosmider B et al (2012) Nrf2 protects human alveolar epithelial cells against injury induced by influenza A virus. Respir Res. https://doi.org/10.1186/1465-9921-13-43

27. Olagnier D et al (2020) SARS-CoV2-mediated suppression of NRF2-signaling reveals potent antiviral and anti-inflammatory activity of 4-octyl-itaconate and dimethyl fumarate. Nat Commun. https://doi.org/10.1038/s41467-020-18764-3

28. Capone F, Ferraro E, Motolese F, Di Lazzaro V (2020) COVID19 in multiple sclerosis patients treated with dimethyl fumarate. J Neurol 1:3. https://doi.org/10.1007/s00415-021-10446-4

29. Singer BA (2017) The role of natalizumab in the treatment of multiple sclerosis: benefits and risks. Ther Adv Neurol Disord. https://doi.org/10.1177/1756285617716002

30. Rommer PS et al (2019) Immunological aspects of approved MS therapeutics. Front Immunol. https://doi.org/10.3389/fimmu. 2019.01564

31. Ellul MA et al (2020) Neurological associations of COVID-19. Lancet Neurol. https://doi.org/10.1016/S1474-4422(20)30221-0

32. Hartung HP, Aktas O, Boyko AN (2015) Alemtuzumab: A new therapy for active relapsing-remitting multiple sclerosis. Mult Scler J. https://doi.org/10.1177/1352458514549398

33. Havrdova E, Cohen JA, Horakova D, Kovarova I, Meluzinova E (2017) Understanding the positive benefit: risk profile of alemtuzumab in relapsing multiple sclerosis: perspectives from the alemtuzumab clinical development program. Ther Clin Risk Manag. https://doi.org/10.2147/TCRM.S143509

34. Coles AJ et al (2012) Alemtuzumab for patients with relapsing multiple sclerosis after disease-modifying therapy: a randomised controlled phase 3 trial. Lancet. https://doi.org/10. 1016/S0140-6736(12)61768-1

35. Coles AJ, Compston DA, Selmaj KW, Lake SL, Moran S, Margolin DH, Norris K, Tandon PK (2008) Alemtuzumab vs. interferon beta-1a in early multiple sclerosis. N Engl J Med 359:1786-1801. https://doi.org/10.1056/nejmoa0802670

36. Wray $S$ et al (2019) Infection risk with alemtuzumab decreases over time: pooled analysis of 6-year data from the CAMMS223, CARE-MS I, and CARE-MS II studies and the CAMMS03409 extension study. Mult Scler J. https://doi.org/10.1177/13524 58518796675

37. Guevara C, Villa E, Cifuentes M, Naves R, de Grazia J (2020) Mild COVID-19 infection in a patient with multiple sclerosis and severe depletion of T-lymphocyte subsets due to alemtuzumab. Mult Scler Relat Disord 44:102314. https://doi.org/10. 1016/j.msard.2020.102314

38. Fernández-Díaz E, Gracia-Gil J, García-García JG, Palao M, Romero-Sánchez CM, Segura T (2020) COVID-19 and multiple sclerosis: a description of two cases on alemtuzumab. Mult Scler Relat Disord 45:102402. https://doi.org/10.1016/j.msard. 2020.102402

39. Fiorella C, Lorna G (2020) COVID-19 in a multiple sclerosis (MS) patient treated with alemtuzumab: insight to the immune 
response after COVID. Mult Scler Relat Disord 46:102447. https://doi.org/10.1016/j.msard.2020.102447

40. Vernengo FF et al (2020) $\mathrm{Cd} 8+\mathrm{t}$ cell immunity is compromised by anti-cd20 treatment and rescued by interleukin-17a. MBio. https://doi.org/10.1128/mBio.00447-20

41. Toubi $E$ et al (2007) Changes in macrophage function after rituximab treatment in patients with rheumatoid arthritis. Ann Rheum Dis. https://doi.org/10.1136/ard.2006.062505

42. Meca-Lallana V, Aguirre C, Río B, Cardeñoso L, Alarcon T, Vivancos J (2020) COVID-19 in 7 multiple sclerosis patients in treatment with ANTI-CD20 therapies. Mult Scler Relat Disord. https://doi.org/10.1016/j.msard.2020.102306

43. Conte WL (2020) Attenuation of antibody response to SARS$\mathrm{CoV}-2$ in a patient on ocrelizumab with hypogammaglobulinemia. Mult Scler Relat Disord. https://doi.org/10.1016/j.msard. 2020.102315

44. Simpson-Yap S et al (2020) First results of the COVID-19 in MS global data sharing initiative suggest anti-CD20 DMTS are associated with worse COVID-19 outcomes. Mult Scler J 26:48-49

45. Avouac J et al (2021) COVID-19 outcomes in patients with inflammatory rheumatic and musculoskeletal diseases treated with rituximab: a cohort study. Lancet Rheumatol. https://doi.org/10. 1016/S2665-9913(21)00059-X

46. Stuve O et al (2019) Effects of cladribine tablets on lymphocyte subsets in patients with multiple sclerosis: an extended analysis of surface markers. Ther Adv Neurol Disord. https://doi.org/10. $1177 / 1756286419854986$

47. Hermann R, Karlsson MO, Novakovic AM, Terranova N, Fluck M, Munafo A (2019) The clinical pharmacology of cladribine tablets for the treatment of relapsing multiple sclerosis. Clin Pharmacokinet. https://doi.org/10.1007/s40262-018-0695-9

48. Wang Y et al (2020) Remdesivir in adults with severe COVID-19: a randomised, double-blind, placebo-controlled, multicentre trial. Lancet. https://doi.org/10.1016/S0140-6736(20)31022-9

49. Lan J et al (2020) Structure of the SARS-CoV-2 spike receptorbinding domain bound to the ACE2 receptor. Nature. https://doi. org/10.1038/s41586-020-2180-5

50. Schlake T, Thess A, Fotin-Mleczek M, Kallen KJ (2012) Developing mRNA-vaccine technologies. RNA Biol. https://doi.org/10. 4161/rna.22269

51. Du L et al (2008) Intranasal vaccination of recombinant adenoassociated virus encoding receptor-binding domain of severe acute respiratory syndrome coronavirus (SARS-CoV) spike protein induces strong mucosal immune responses and provides longterm protection against SARS-. J Immunol. https://doi.org/10. 4049/jimmunol.180.2.948

52. Lebrun C, Vukusic S (2019) Immunization and multiple sclerosis: Recommendations from the French multiple sclerosis society. Mult Scler Relat Disord. https://doi.org/10.1016/j.msard.2019.04. 004

53. Mehling $M$ et al (2013) Preserved antigen-specific immune response in patients with multiple sclerosis responding to IFN $\beta$ therapy. PLoS ONE. https://doi.org/10.1371/journal.pone.00785 32

54. Olberg HK, Cox RJ, Nostbakken JK, Aarseth JH, Vedeler CA, Myhr KM (2014) Immunotherapies influence the influenza vaccination response in multiple sclerosis patients: an explorative study. Mult Scler J. https://doi.org/10.1177/1352458513513970

55. Olberg HK et al (2018) Antibody response to seasonal influenza vaccination in patients with multiple sclerosis receiving immunomodulatory therapy. Eur J Neurol. https://doi.org/10.1111/ene. 13537

56. Metze $\mathrm{C}$ et al (2019) Immunogenicity and predictors of response to a single dose trivalent seasonal influenza vaccine in multiple sclerosis patients receiving disease-modifying therapies. CNS Neurosci Ther. https://doi.org/10.1111/cns.13034

57. Bar-Or A et al (2013) Teriflunomide effect on immune response to influenza vaccine in patients with multiple sclerosis. Neurology $81: 552-558$

58. Bar-Or A et al (2015) Randomized study of teriflunomide effects on immune responses to neoantigen and recall antigens. Neurol Neuroimmunol NeuroInflammation. https://doi.org/10.1212/NXI. 0000000000000070

59. Von Hehn $\mathrm{C}$ et al (2018) Immune response to vaccines is maintained in patients treated with dimethyl fumarate. Neurol Neuroimmunol NeuroInflammation. https://doi.org/10.1212/NXI.00000 00000000409

60. Kappos L et al (2015) Randomized trial of vaccination in fingolimod-treated patients with multiple sclerosis. Neurology. https:// doi.org/10.1212/WNL.0000000000001302

61. Ufer $\mathrm{M}$ et al (2017) Impact of siponimod on vaccination response in a randomized, placebo-controlled study. Neurol Neuroimmunol NeuroInflammation. https://doi.org/10.1212/NXI.0000000000 000398

62. Kaufman M, Pardo G, Rossman H, Sweetser MT, Forrestal F, Duda P (2014) Natalizumab treatment shows no clinically meaningful effects on immunization responses in patients with relapsing-remitting multiple sclerosis. J Neurol Sci. https://doi.org/10. 1016/j.jns.2014.03.035

63. McCarthy CL, Tuohy O, Compston DAS, Kumararatne DS, Coles AJ, Jones JL (2013) Immune competence after alemtuzumab treatment of multiple sclerosis. Neurology. https://doi.org/10.1212/ WNL.0b013e3182a35215

64. Sellner J, Rommer PS (2020) Immunological consequences of 'immune reconstitution therapy' in multiple sclerosis: a systematic review. Autoimmun Rev. https://doi.org/10.1016/j.autrev. 2020.102492

65. Bar-Or A et al (2020) Effect of ocrelizumab on vaccine responses in patients with multiple sclerosis: the VELOCE study. Neurology. https://doi.org/10.1212/WNL.0000000000010380

66. Wu AHGF, Boschert U, Hayward B, Lebson LA (2021) Cross evaluating the impact of cladribine tablets on the development of antibody titers: interim results from the CLOCK-MS influenza vaccine substudy. CLOCK-MS study

67. Sanjeev Roy UB (2021) Analysis of influenza and varicella zoster virus vaccine antibody titers in patients with relapsing multiple sclerosis treated with cladribine tablets. MS magnify

68. Giovannoni $\mathrm{G}$ et al (2010) A placebo-controlled trial of oral cladribine for relapsing multiple sclerosis. N Engl J Med. https://doi. org/10.1056/NEJMoa0902533 